\title{
Contrasting trends in rates of genital chlamydial infection and ectopic pregnancy in South East Thames Region, England and Uppsala County, Sweden: ecological study
}

\author{
Nicola Low, Matthias Egger, Ian Simms, Björn Herrmann
}

Sexually transmitted chlamydial infections are the most common preventable cause of pelvic inflammatory disease, and tubal infertility. ${ }^{1}$ In Sweden, falling rates of chlamydia, ${ }^{2}$ pelvic inflammatory disease, ${ }^{2}$ and ectopic pregnancy ${ }^{3}$ have been attributed to intensive testing, treatment and partner notification to detect asymptomatic infections, in a variety of healthcare settings since the 1980s. In Britain, routine surveillance, testing and partner notification for chlamydia currently occur only in genitourinary clinics, although an expert advisory committee of the Chief Medical Officer has now recommended targeted opportunistic screening in other health care settings. ${ }^{4}$ We conducted ecological comparisons of the effects of different levels of chlamydia control on the incidence of one consequence, ectopic pregnancy, in SE Thames Region, England and Uppsala County, Sweden.

\section{Methods}

For SE Thames Region we obtained information from aggregated genitourinary clinic reports of chlamydia and from hospital episode statistics for ectopic pregnancy. For Uppsala, we used data from University Hospital, which deals with $99 \%$ of chlamydia specimens and all ectopic pregnancies in the County. ${ }^{3}$ Ectopic pregnancy was recorded in both settings as International Classification of Diseases, 9th revision, XI.633.

Yearly chlamydia rates per 100000 women and ectopic pregnancies per 1000 pregnancies (total of live births, induced abortions and ectopic pregnancies) for 15-34 year olds were calculated from 1988-1996. Direct standardisation was used to calculate age adjusted summary rates, using SE Thames census enumerated population in 1991 as the reference standard for chlamydia and, for ectopic pregnancy, the number of pregnancies in SE Thames residents in 1991. $\chi^{2}$ Tests for trend were calculated for both conditions. Our analyses were restricted to women aged 15-34 years because, in this range, ectopic pregnancy rates are strongly correlated with rates of chlamydia diagnosed in the same year. ${ }^{3}$

Dr N Low, Department of Social Medicine, University of Bristol, Canynge Hall, Whiteladies Road, Bristol BS8 2PR.

Accepted for publication 3 March 1999

\section{Results}

From 1988-1996, in SE Thames Region, 14420 diagnoses of chlamydia and 536854 pregnancies (5295 ectopic) in women aged 15-34 years were recorded. In Uppsala County there were 3739 episodes of chlamydia and 38533 pregnancies (505 ectopic).

The respective 1991 populations were 558460 and 39 923. Figure 1 shows that, over the study period, age adjusted rates of chlamydia cases per 100000 women declined by $67 \%$, from 1701 (95\% confidence intervals 1574 to 1828 ) to 560 (490 to 630) in Uppsala and increased by $76 \%$, from 245 (232 to 257 ) to 430 (412 to 448) in SE Thames. Rates of ectopic pregnancy per 1000 total pregnancies fell $56 \%$, from 18 (14 to 22 ) to 8 (5 to 11 ) in Uppsala, and rose $39 \%$, from 7 (6 to 8 ) to 10 (9 to 11) in SE Thames.

\section{Comment}

Intensive chlamydia control activities in Uppsala, Sweden are associated with marked falls in chlamydia and ectopic pregnancy rates in women under 35 years. During the same nine year period rates of both conditions rose in SE Thames Region.

Higher chlamydia rates in Uppsala are probably explained by widespread detection of asymptomatic cases, with only a quarter of specimens coming from genitourinary clinics. ${ }^{5}$ By 1996 however, the chlamydia rate in Uppsala, based on cases diagnosed in all settings was only 1.3 times higher than that in SE Thames, which only includes cases from genitourinary clinics. Reasons for the initial difference in ectopic pregnancy rates are less clear. The diagnosis of ectopic pregnancy is well defined but case reporting may be less accurate and complete in SE Thames. Extensive use of laparoscopy in Sweden, detecting some early ectopic pregnancies that would have aborted spontaneously, may exaggerate this disparity. The opposing direction of trends in the two countries is, however, more important than absolute rates for postulating the effects of chlamydia control policies.

The decline in chlamydia rates in Uppsala is now slowing down, ${ }^{5}$ so the achievable limit of the strategies adopted may have been reached. The recommendations of the Chief Medical Officer's report ${ }^{4}$ share features of the Swedish approach, but are less comprehensive. Opportunistic screening of sexually active women under 25 years and other selected groups is proposed, primarily in 

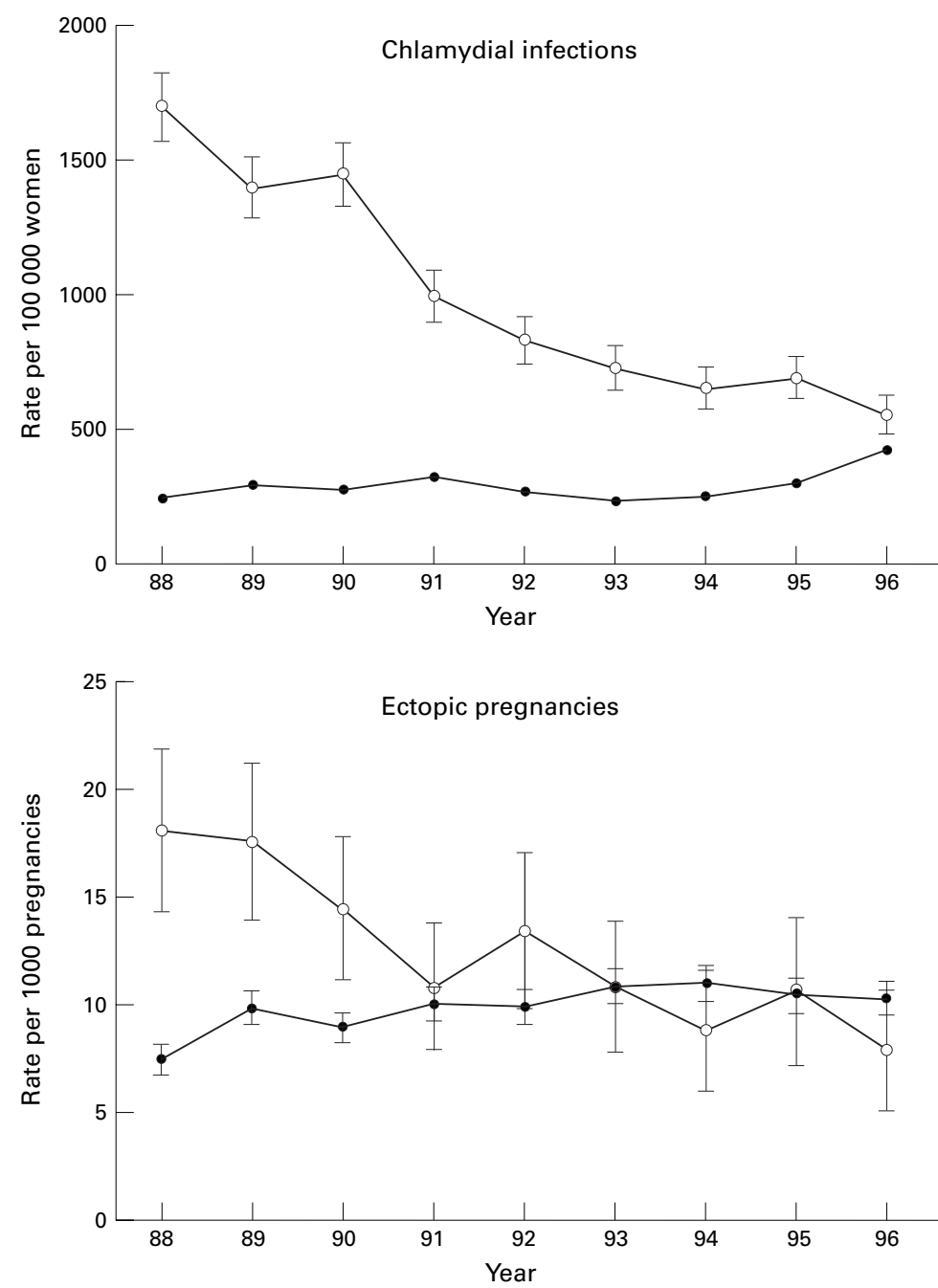

Figure 1 Age standardised rates, with 95\% confidence intervals, of genital chlamydia per 100000 women (top panel) and ectopic pregnancy per 1000 pregnancies (lower panel), reported among women aged 15 to 34 years in SE Thames Region, England and Uppsala County, Sweden, 1988 to 1996. SE Thames rates represented by solid circles, Uppsala rates represented by open circles. Error bars represent $95 \%$ confidence intervals. Error bars around the chlamydia rates in SE Thames are present but are not visible because the confidence intervals are narrow. $\chi^{2}$ Tests for trend in rates of chlamydia and ectopic pregnancy in SE Thames Region and Uppsala County all significant at $p<0.001$. general practice and family planning clinics, with partner notification undertaken in genitourinary clinics. Policy makers in Britain should consider whether more comprehensive chlamydia control measures such as, the widespread use of improved diagnostic tests, partner notification in non-genitourinary settings, case finding in men and register-based screening are required.

Chlamydia surveillance should be extended to cases diagnosed in non-genitourinary settings and the value of ectopic pregnancy rates in women under 35 years as a marker of the effectiveness of screening should be further investigated.

An earlier version of this paper was presented at the Medical Society for the Study of Venereal Diseases Spring Meeting, Oxford, 3-5 July 1997.

We thank staff at the Communicable Disease Surveillance Centre, South Thames Information Management and Technology Centre and Office for National Statistics, England for providing data for SE Thames Region and the County Statistical Office and Epidemiological Centre, Uppsala and National Board of Health and Welfare, Stockholm for data about Uppsala.

The Department of Social Medicine at the University of Bristol is part of the MRC Health Services Research Collaboration.

Nicola Low designed the study, performed statistical analyses and drafted the paper. Matthias Egger contributed to statistical analysis and writing the paper. Björn Herrmann and Ian Simms provided data for Uppsala and SE Thames Region, respectively, and contributed to writing the paper.

Funding: none.

Conflicts of interest: none.

1 Cates W Jr, Rolfs RT Jr, Aral SO. Sexually transmitted diseases, pelvic inflammatory disease, and infertility: an epidemiologic update. Epidemiol Rev 1990;12:199-220.

2 Kamwendo F, Forslin L, Bodin L, et al. Decreasing incidences of gonorrhea- and chlamydia-associated pelvic inflammatory disease: a 25 -year study from an urban area of central Sweden. Sex Transm Dis 1996;23:384-91.

3 Egger M, Low N, Davey Smith G, et al. Screening for chlamydial infections and the risk of ectopic pregnancy in a county in Sweden: ecological analysis. BMF 1998;316: county in

4 CMO's Expert Advisory Group on Chlamydia trachomatis. London: Department of Health, 1998.

5 Herrmann B, Egger M. Genital Chlamydia trachomatis infections in Uppsala County, Sweden, 1985-1993: declining rates for how much longer? Sex Transm Dis 1995;22: 253-60. 